\title{
Clinical Features and Risk Factors of Pulmonary Hypertension in Chinese Patients With Systemic Lupus Erythematosus
}

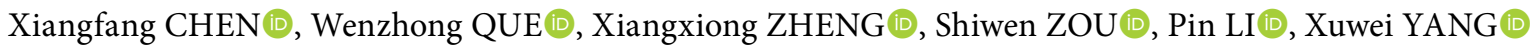 \\ Department of Rheumatology, Fujian Medical University Union Hospital, Fuzhou, China
}

\begin{abstract}
Objectives: This study aims to evaluate the systemic lupus erythematosus (SLE) frequency, clinical characteristics, and laboratory features of pulmonary hypertension (PH) in a Chinese population with SLE and to evaluate the risk factors contributing in early diagnosis.

Patients and methods: A total of 39 patients ( 2 males, 37 females; mean age $38.2 \pm 14.9$ years; range, 16 to 71 years) with combined SLE and PH and 407 patients (43 males, 364 females; mean age $34.8 \pm 14.0$ years; range, 7 to 73 years) with SLE but without PH (NonPH) were enrolled and categorized into two groups, namely, $\mathrm{PH}$ and NonPH groups. The demographic and clinical characteristics of all patients, including disease duration, comorbidity, malar rash, epilepsy, arthritis, oral ulcer, photosensitivity, Raynaud's phenomenon, serositis, dyspnea, and visceral damage, were recorded. Laboratory parameters, including blood and urine routine, biochemical markers, 24-hour proteinuria, plasma N-terminal pro-brain natriuretic peptide (NT-proBNP), immunoglobulin, complement 3 and 4, and autoantibodies, were tested. Inflammatory indexes, such as erythrocyte sedimentation rate and C-reactive protein level, were collected. Disease activity was assessed with systemic lupus erythematosus disease activity index score. Organ damage was assessed with the use of Systemic Lupus International Collaborating Clinics/American College of Rheumatology (SLICC/ACR) Damage Index (SLICC/ACR-DI; SDI). Pulmonary arterial systolic pressure was measured using echocardiography.

Results: Pulmonary hypertension frequency in our Chinese population with SLE was $8.74 \%$. Statistical differences in neuropsychological symptoms, epilepsy, serositis, Raynaud's phenomenon, tachypnea, albumin, creatinine, abnormal electrocardiograph, urine protein, urine red blood cell rate, NT-proBNP, body mass index, SDI and duration of SLE were found between SLE patients with or without PH. Multivariate logistic regression revealed that Raynaud's phenomenon, serositis, tachypnea, epilepsy, and positive anti-U1 small nuclear ribonucleoprotein (U1RNP) were significant risk factors for PH in SLE.

Conclusion: Chinese patients with SLE are at high risk for PH if they present with epilepsy, Raynaud's phenomenon, serositis, tachypnea, and positive anti-U1RNP. Echocardiography is highly recommended to be performed on Chinese patients with SLE for the screening of PH.

Keywords: Hypertension; lupus erythematosus; pulmonary; risk factors; systemic.
\end{abstract}

Pulmonary hypertension $(\mathrm{PH})$ is a serious condition that may ultimately lead to death if left untreated. In 2013, PH was classified into five groups at the Fifth World Symposium on PH held in Nice, France. ${ }^{1}$ Connective tissue diseaseassociated pulmonary arterial hypertension (CTD-APAH) belongs to group 1. Given the epidemiological difference in connective tissue diseases (CTDs) between China and Western countries, systemic lupus erythematosus (SLE) acquires the largest portion of CTD-APAH in China, whereas systemic sclerosis constitutes the major portion of CTD-APAH in Western countries. ${ }^{2,3} \mathrm{PH}$ is a serious complication of SLE; however, this condition is rarely reported. Although the prevalence of $\mathrm{PH}$ in SLE was estimated to be different worldwide because of the lack of a uniform $\mathrm{PH}$ definition or different diagnostic approaches, ${ }^{4}$ patients with combined SLE and PH exhibit lower one-year

Received: December 13, 2017 Accepted: April 24, 2018 Published online: August 16, 2018

Correspondence: Xuwei Yang, MD. Department of Rheumatology, Fujian Medical University Union Hospital, 350001 Fuzhou, China. Tel: 008659183357896 e-mail:yangxwhk@126.com 
survival rate and quality of life than those of idiopathic patients, ${ }^{5}$ particularly for Chinese people with increased prevalence rate. Early diagnosis and treatment are necessary to improve abnormal pulmonary and cardiac functions ${ }^{6}$ and even decrease morbidity and mortality of patients with SLE and PH. ${ }^{7}$ Factors involved in the pathogenesis of SLE-associated $\mathrm{PH}$ are genetic predisposition, environmental stimuli, and immune system dysfunction, ${ }^{4}$ which make prognosis during progression possible.

Right heart catheterization (RHC) is the gold standard for the diagnosis of $\mathrm{PH}$, but adverse events may exist after catheterization. ${ }^{8}$ Hence, as a kind of noninvasive procedure, echocardiography is of considerable value for $\mathrm{PH}$ in clinical practice. Echocardiography provides an estimate of right ventricular function and pulmonary artery pressure and is useful in ruling out secondary causes of $\mathrm{PH}$, such as left heart disease and congenital heart disease. ${ }^{9}$ Pulmonary arterial systolic pressure (PASP) values of $\geq 40 \mathrm{mmHg}$ by echocardiogram were recommended for screening patients with SLE, who have suspected $\mathrm{PH} .{ }^{10}$

In this study, we aimed to evaluate the SLE frequency, clinical characteristics, and laboratory features of $\mathrm{PH}$ in a Chinese population with SLE and to evaluate the risk factors contributing in early diagnosis.

\section{PATIENTS AND METHODS}

Medical records of 446 patients (46 males, 400 females; mean age 35.1 14.1 years; range, 7 to 73 years) with SLE were collected in the Department of Rheumatology, Fujian Medical University Union Hospital between January 2013 and March 2017 according to Systemic Lupus International Collaborating Clinics (SLICC) 2012 classification criteria. ${ }^{11}$ Among them, 39 patients ( 2 males, 37 females; mean age $38.2 \pm 14.9$ years; range, 16 to 71 years) passed the SLICC 2012 classification criteria of SLE and the PH criteria previously described and were recruited as test subjects (PH group), while the remaining 407 patients (43 males, 364 females; mean age $34.8 \pm 14.0$ years; range, 7 to 73 years) were recruited as controls (NonPH group). The $\mathrm{PH}$ group consisted of patients with PASP of $\geq 40 \mathrm{mmHg}$ by echocardiography, ${ }^{10,12}$ while the NonPH group consisted of patients with PASP of $<40 \mathrm{mmHg}$. Patients with SLE were excluded if they suffered from diseases that could affect pulmonary artery pressure, including overlapping syndrome, interstitial lung disease, chronic thromboembolic $\mathrm{PH}$, congenital heart diseases, significant valvular disease, chronic obstructive pulmonary disease, and pregnancy. The study protocol was approved by the Fujian Medical University Union Hospital Ethics Committee (No.2018KY015). A written informed consent was obtained from each patient. The study was conducted in accordance with the principles of the Declaration of Helsinki.

We recorded the demographic and clinical characteristics of all patients, including disease duration, comorbidity, malar rash, epilepsy, arthritis, oral ulcer, photosensitivity, Raynaud's phenomenon, serositis, dyspnea, and visceral damage. We also tested laboratory parameters, such as blood and urine routine, biochemical markers, 24-hour proteinuria, plasma $\mathrm{N}$-terminal pro-brain natriuretic peptide (NT-proBNP), immunoglobulin, complement 3 and 4 , and autoantibodies (such as anti-doublestranded deoxyribonucleic acid, anti-Sjögren's syndrome type A (anti-SSA), anti-Sjögren's syndrome type B (anti-SSB), anti-Sm, anti-U1 small nuclear ribonucleoprotein (U1RNP), anticardiolipin antibodies, and $\beta 2$-glycoprotein I). Inflammatory indices, including erythrocyte sedimentation rate (ESR) and C-reactive protein (CRP) level, were also obtained. Disease activity was assessed using systemic lupus erythematosus disease activity index (SLEDAI) score ${ }^{13}$ and organ damage was evaluated with Systemic Lupus International Collaborating Clinics/American College of Rheumatology (SLICC/ACR) Damage Index (SLICC/ACR-DI; $\mathrm{SDI}) .{ }^{14}$ All laboratory data were measured in the Department of Laboratory Medicine, Fujian Medical University Union Hospital. PASP was measured by specialized echocardiographers in the Department of Cardiology of the same hospital using GE Vivid E9 Ultrasound System (General Electric Company, Horten, Norway). Confirmation of PASP by a designated echocardiographer was required if the initial PASP was $\geq 40 \mathrm{mmHg}$. 


\section{Statistical analysis}

Data distribution was tested by ShapiroWilk method. Results were reported as mean \pm standard deviation or number (percentage). Non-normally distributed data or ranked data were compared between groups using the Mann-Whitney U test. Categorical data were compared with Chi-square test or Fisher's exact test. Multivariate logistic regression was adopted to report the correlation between the variables and $\mathrm{PH}$ in all patients with SLE. Data analysis was performed using PASW version 17.0 for Windows (SPSS Inc., Chicago. IL. USA). A difference was considered significant if the twoside $p$ value was less than 0.05 .

\section{RESULTS}

In terms of demographic data (age, sex), no significant difference was found between the two groups except body mass index (BMI). The PH group showed significantly increased frequencies of neuropsychological symptom, epilepsy, serositis, Raynaud's phenomenon, BMI, and tachypnea, as well as extended illness duration and increased SDI in clinical features (Table 1). In particular, more patients from the $\mathrm{PH}$ group manifested abnormal electrocardiography (Table 2). In the laboratory test, the PH group exhibited decreased albumin level and increased creatinine level, positive proteinuria rate, positive urine red blood cell rate, and NT-proBNP ratio.

Table 1. Demographic and clinical characteristics in systemic lupus erythematosus with or without pulmonary hypertension

\begin{tabular}{|c|c|c|c|c|c|c|c|}
\hline \multirow[t]{2}{*}{ Characteristics } & \multicolumn{3}{|c|}{ SLE-PH $(n=39)$} & \multicolumn{3}{|c|}{ SLE-NonPH $(n=407)$} & \multirow[b]{2}{*}{$p$} \\
\hline & $\mathrm{n}$ & $\%$ & Mean \pm SD & $\mathrm{n}$ & $\%$ & Mean \pm SD & \\
\hline Age (year) & & & $38.2 \pm 14.0$ & & & $34.8 \pm 14.0$ & 0.234 \\
\hline $\begin{array}{l}\text { Gender } \\
\text { Female }\end{array}$ & 37 & 94.9 & & 363 & 89.2 & & 0.401 \\
\hline Body mass index $\left(\mathrm{kg} / \mathrm{m}^{2}\right)$ & & & $23.5 \pm 4.3$ & & & $21.5 \pm 3.4$ & 0.043 \\
\hline Systemic lupus erythematosus duration (year) & & & $5.7 \pm 7.0$ & & & $3.5 \pm 5.0$ & 0.042 \\
\hline Pulmonary arterial systolic pressure $(\mathrm{mmHg})$ & & & $54.2 \pm 18.2$ & & & $27.5 \pm 5.8$ & $<0.001$ \\
\hline Malar rash & 17 & 43.6 & & 170 & 42.0 & & 0.845 \\
\hline Epilepsy & 3 & 7.9 & & 7 & 1.7 & & 0.046 \\
\hline Fever & 13 & 33.3 & & 136 & 33.6 & & 0.975 \\
\hline Arthritis & 19 & 48.7 & & 221 & 54.4 & & 0.494 \\
\hline Photosensitivity & 4 & 10.3 & & 55 & 13.6 & & 0.559 \\
\hline Serositis & 26 & 66.7 & & 121 & 29.9 & & $<0.001$ \\
\hline Psychiatricsymptoms & 5 & 12.8 & & 12 & 3.0 & & 0.009 \\
\hline Myalgia/myasthenia & 1 & 2.6 & & 6 & 1.5 & & 0.477 \\
\hline Xerostomia/xeroma & 3 & 7.7 & & 30 & 7.5 & & 1.000 \\
\hline Oral ulcer & 6 & 15.4 & & 44 & 10.9 & & 0.561 \\
\hline Raynaud's phenomenon & 12 & 30.8 & & 38 & 9.4 & & $<0.001$ \\
\hline Alopecia & 6 & 15.8 & & 49 & 12.3 & & 0.722 \\
\hline Fingertip vasculitis & 2 & 5.1 & & 14 & 3.5 & & 0.932 \\
\hline Peripheral neuritis & 1 & 2.6 & & 4 & 1 & & 0.371 \\
\hline Erythra & 15 & 38.5 & & 125 & 30.9 & & 0.329 \\
\hline Tachypnea & 19 & 52.8 & & 52 & 13.2 & & $<0.001$ \\
\hline Livedo reticularis & 0 & 0 & & 3 & 0.7 & & - \\
\hline SLEDAI & & & $10.9 \pm 7.1$ & & & $7.8 \pm 5.3$ & 0.050 \\
\hline SDI & & & $2.5 \pm 1.9$ & & & $0.6 \pm 0.8$ & $<0.001$ \\
\hline
\end{tabular}


Table 2. Laboratory findings in systemic lupus erythematosus with or without pulmonary hypertension

\begin{tabular}{|c|c|c|c|c|c|c|c|}
\hline \multirow[t]{2}{*}{ Characteristics } & \multicolumn{3}{|c|}{ SLE-PH (n=39) } & \multicolumn{3}{|c|}{ SLE-NonPH $(n=407)$} & \multirow[b]{2}{*}{$p$} \\
\hline & $\mathrm{n}$ & $\%$ & Mean \pm SD & $\mathrm{n}$ & $\%$ & Mean \pm SD & \\
\hline Anti-dsDNA Ab & & & $35.3 \pm 36.6$ & & & $30.6 \pm 35.2$ & 0.729 \\
\hline ANCA & 1 & 4.2 & & 7 & 2.5 & & 0.491 \\
\hline $\mathrm{RF}$ & 9 & 50.0 & & 86 & 35.7 & & 0.224 \\
\hline ANA & 33 & 100 & & 364 & 99.2 & & - \\
\hline Spot nucleus & 22 & 66.7 & & 229 & 64.9 & & 0.836 \\
\hline Anti-U1RNP Ab & 21 & 63.6 & & 174 & 47.8 & & 0.081 \\
\hline Anti-SM Ab & 13 & 39.4 & & 123 & 33.6 & & 0.502 \\
\hline Anti-SSA Ab & 18 & 54.5 & & 251 & 68.2 & & 0.110 \\
\hline Anti-SSB Ab & 8 & 24.2 & & 92 & 25.1 & & 0.910 \\
\hline Anti-SCL-70 Ab & 0 & 0 & & 4 & 1.4 & & - \\
\hline Anti-PM-SCL Ab & 2 & 6.1 & & 12 & 3.3 & & 1.000 \\
\hline Anti-JO-1 Ab & 0 & & & 0 & & & - \\
\hline Anti-Centromere Ab & 1 & 3.0 & & 11 & 3.1 & & 1.000 \\
\hline Anti-Proliferation $\mathrm{Ab}$ & 0 & & & 9 & 2.5 & & - \\
\hline Anti-Nucleosomes Ab & 20 & 60.6 & & 185 & 50.5 & & 0.268 \\
\hline Anti-Histone Ab & 16 & 48.5 & & 141 & 39.0 & & 0.278 \\
\hline Anti-rRNP Ab & 14 & 42.4 & & 146 & 39.9 & & 0.776 \\
\hline Anti-Mitochondrial Ab & 3 & 9.4 & & 58 & 15.9 & & 0.970 \\
\hline Anti-Cardiolipin IgM Ab & 1 & 3.4 & & 21 & 6.7 & & 0.778 \\
\hline Anti-Cardiolipin IgG Ab & 5 & 17.9 & & 67 & 21.4 & & 0.659 \\
\hline Anti- $\beta 2-G P 1 \mathrm{Ab}$ & 3 & 10.7 & & 54 & 17.7 & & 0.498 \\
\hline Abnormal ECG & 25 & 75.8 & & 216 & 57.6 & & 0.042 \\
\hline Leukopenia & 13 & 36.1 & & 151 & 37.8 & & 0.846 \\
\hline Anemia & 26 & 72.2 & & 247 & 61.9 & & 0.220 \\
\hline Thrombocytopenia & 10 & 27.8 & & 102 & 25.6 & & 0.778 \\
\hline Urine protein & 35 & 92.1 & & 259 & 66.7 & & 0.001 \\
\hline Urine RBC & 23 & 60.5 & & 149 & 39.5 & & 0.009 \\
\hline $\operatorname{ALB}(\mathrm{g} / \mathrm{L})$ & & & $26.0 \pm 6.9$ & & & $30.9 \pm 7.6$ & $<0.001$ \\
\hline GLB (g/L) & & & $33.3 \pm 10.5$ & & & $34.9 \pm 8.8$ & 0.145 \\
\hline CRE (umol/L) & & & $166.1 \pm 219.7$ & & & $69.6 \pm 74.6$ & $<0.001$ \\
\hline LDH (IU/L) & & & $307.7 \pm 256.5$ & & & $265.1 \pm 186.4$ & 0.116 \\
\hline $\operatorname{IgG}(\mathrm{g} / \mathrm{L})$ & & & $15.5 \pm 8.5$ & & & $17.2 \pm 7.9$ & 0.051 \\
\hline $\operatorname{IgM}(\mathrm{g} / \mathrm{L})$ & & & $1.1 \pm 0.7$ & & & $1.3 \pm 1.0$ & 0.064 \\
\hline $\operatorname{IgA}(\mathrm{g} / \mathrm{L})$ & & & $2.5 \pm 1.3$ & & & $2.8 \pm 1.4$ & 0.241 \\
\hline C3 (g/L) & & & $0.5 \pm 0.3$ & & & $0.5 \pm 0.3$ & 0.953 \\
\hline C4 (g/L) & & & $0.1 \pm 0.1$ & & & $0.1 \pm 0.1$ & 0.136 \\
\hline $\mathrm{ESR}(\mathrm{mm} / \mathrm{h})$ & & & $54.9 \pm 35.4$ & & & $50.5 \pm 39.9$ & 0.266 \\
\hline CRP & 14 & 54.8 & & 98 & 28.7 & & 0.055 \\
\hline NT-proBNP & 15 & 93.8 & & 21 & 53.8 & & 0.005 \\
\hline
\end{tabular}

SLE: Systemic lupus erythematosus; PH: Pulmonary hypertension; SD: Standard deviation; Anti-dsDNA: Anti-double-stranded deoxyribonucleic acid; Ab: Antibody; ANCA: Antineutrophil cytoplasmic antibodies; RF: Rheumatoid factor; ANA: Antinuclear antibody; Anti-U1RNP: Anti-U1 small nuclear ribonucleoprotein; Anti-SM: Anti-Smith; Anti-SSA: anti-Sjögren's syndrome type A; Anti-SSB: Anti-Sjögren's syndrome type B; Anti-SCL-70: Antitopoisomerase; Anti-PM-SCL: Anti- polymyositis-sclerosis; Anti-JO-1: Anti-histidyl-tRNA synthetase; Anti-rRNP: Anti- ribosomal ribonucleoprotein; Anti- 32 GP1: Anti-ß2-glycoprotein I; ECG: Echocardiography; RBC: Red blood cell; ALB: Albumin; GLB: Globulin; CRE: Creatinine; LDH: Lactate dehydrogenase; Ig: Immunoglobulin; C: Complement: ESR: Erythrocyte sedimentation rate; CRP: C-reactive protein; NT-proBNP: N-terminal pro-brain natriuretic peptide. 


\begin{tabular}{|lccc|}
\hline \multicolumn{4}{l}{$\begin{array}{l}\text { Table 3. Significant factors for pulmonary hypertension in systemic } \\
\text { lupus erythematosus observed in multivariate logistic regression }\end{array}$} \\
\hline Variables & Odds ratio & $95 \%$ CI & $p$ \\
\hline Raynaud's phenomenon & 39.208 & $6.143-250.244$ & $<0.001$ \\
Serositis & 10.144 & $1.771-58.108$ & 0.009 \\
Tachypnea & 12.539 & $2.996-53.019$ & 0.001 \\
Epilepsy & 21.353 & $1.725-264.342$ & 0.017 \\
Anti-U1RNP & 6.117 & $1.390-26.921$ & 0.017 \\
\hline CI: Confidence interval; U1RNP: Nuclear ribonucleoprotein. & \\
\hline
\end{tabular}

No significant difference was found in items related to anti-double-stranded deoxyribonucleic acid antibody, ESR, CRP, immunoglobulin M, immunoglobulin $\mathrm{G}$, complement 3 , hemogram test, anti-U1RNP antibody, anti-SM antibody, anti-SSA antibody, anti-SSB antibody, antineutrophil cytoplasmic antibodies, or SLEDAI (Table 2).

Multivariate logistic regression method was used to determine the correlation between SLE-associated $\mathrm{PH}$ and the factors, such as serositis, Raynaud's phenomenon, tachypnea, antiU1RNP, duration of SLE, and epilepsy. Among the above factors, Raynaud's phenomenon, serositis, tachypnea, epilepsy, and positive anti-U1RNP were significantly associated with the occurrence of PH in SLE (Table 3).

\section{DISCUSSION}

Pulmonary hypertension prevalence in SLE varied widely between $0.5 \%$ and $17.5 \% .{ }^{4}$ In 2014 , $\mathrm{PH}$ prevalence in Chinese patients with SLE was 3.8\% according to the Chinese SLE Treatment and Research (CSTAR) group registry. ${ }^{15}$ The accuracy limitation of echocardiography and small sampling size may be responsible for the increased frequency in this study. ${ }^{16,17}$ SLE-associated $\mathrm{PH}$ belongs to the CTD-APAH category, ${ }^{1,18}$ because SLE is a common underlying CTD with PH complication as the Registry to Evaluate Early and Longterm (REVEAL) Pulmonary Arterial Hypertension Disease Management had reported. ${ }^{19}$ Patients with SLE were extremely young at the time of $\mathrm{PH}$ diagnosis. ${ }^{2}$ Therefore, an in-depth investigation of the features of SLE-associated PH is extremely important.
Consistent with findings in previous reports, increased positive percentages of serositis and Raynaud's phenomenon concurrent with extended illness duration and increased SDI score in $\mathrm{PH}$ group were observed in our study. ${ }^{20,21}$ Multiple organs were injured during the course of SLE progression. Thus, increased pulmonary vascular resistance (PVR) plays an important role in $\mathrm{PH}$ development. ${ }^{22}$ Numerous factors, such as extensive endothelial, adventitial, and smooth muscle dysfunction, vasodilator and vasoconstrictor imbalance, inflammatory and uncontrolled immune response, and an imbalance between proliferation and apoptosis, are involved to result in the increased PVR in patients with SLE. ${ }^{23,24}$ Increased pulmonary capillary resistance and hypoxia causes PVR increase when the lung is injured. Pulmonary arterial vasoconstriction, which is caused by general vascular disorder, occurs when extensive connective tissues are invaded in patients with SLE. Raynaud's phenomenon and increased anti-U1RNP and anti-SSA levels are clearly observed..$^{40,25}$ However, anti-SSA levels showed no significant difference between $\mathrm{PH}$ and NonPH groups possibly because of sampling error and small sample size.

Owing to autoimmunity, renal function is impaired when kidneys are involved in SLE. ${ }^{26}$ As a common comorbidity and independent risk factor for poor outcome in SLE, the damage is intensified by $\mathrm{PH} .{ }^{27}$ Impaired renal function and increased NT-proBNP are indicative that the right heart function is affected. Certain researchers believed that deterioration of these factors is related to the progression of cardiopulmonary involvement resulting from PH. ${ }^{28,29}$ Increased PVR aggravated cardiac preload in $\mathrm{PH}$ and electrical activity of the heart consequently changed. ${ }^{30}$ Data 
from this study also confirmed that the injury to kidneys and heart is more serious in the $\mathrm{PH}$ group compared with the NonPH group.

Several researchers presented that disease duration, Raynaud's phenomenon, pleuritis, positive anti-U1RNP antibody, positive anti-SSA antibody, and positive anti-cardiolipin antibodies are independent predictors of $\mathrm{PH}$ in SLE. ${ }^{15,21}$ We found that tachypnea, which has been rarely investigated, is an important marker relevant to $\mathrm{PH}$ in SLE. Dyspnea is one of the cardinal clinical manifestations of PH. No difference in dyspnea percentage was observed between idiopathic PAH and SLE-APAH. ${ }^{30}$ Considering the exclusion of the lung and heart diseases affecting pulmonary artery pressure in our study, tachypnea was considered as an excellent indicator of SLEassociated $\mathrm{PH}$ that consumes short time and is assessed and observed easily in the early phase.

Although $\mathrm{PH}$ is a major cause of mortality in SLE, more than $40 \%$ of patients with SLEcombined with early $\mathrm{PH}$ were asymptomatic or slightly symptomatic; thus, $\mathrm{PH}$ is frequently neglected. ${ }^{1,25}$ The gold standard for $\mathrm{PH}$ diagnosis is mean pulmonary artery pressure at rest $\geq 25 \mathrm{mmHg}$ by $\mathrm{RHC}$. However, RHC is not commonly practiced in the rheumatology departments of numerous Chinese hospitals because of its high cost, lack of medical equipment, poor awareness of $\mathrm{PH}$, and fear of severe complications or discomfort from the invasive procedure. Hence, no RHC data were collected in this retrospective study. However, as a non-invasive procedure, regular echocardiography provides information on not only the estimation of pulmonary hemodynamics but also the function of the right atrium. ${ }^{31,32}$ Echocardiography can be used in preliminary screening, ${ }^{10}$ and patients with SLE and with PASP $\geq 40 \mathrm{mmHg}$ should undergo RHC for proper diagnosis. The combination of six-minute walk test, NT-proBNP, clinical findings, and monitoring of pulmonary pressure can aid in the early diagnosis and monitoring of treatment response to treatment. ${ }^{33}$

Patients with SLE benefit considerably if the SLE-associated PH markers are determined effectively. Immune inflammatory factors are involved in the pathogenesis of combined SLE and $\mathrm{PH}$ in addition to vascular medial smooth muscle hypertrophy, intimal proliferation and fibrosis, and adventitial thickening. ${ }^{21,34}$ Inflammation plays an important role in the pathogenesis of $\mathrm{PH}$ in patients with SLE. The use of immunosuppressants, including intravenous cyclophosphamide and glucocorticoids, can achieve improved outcomes for SLE-PH patients. ${ }^{35}$

Immunosuppression and vasodilators produce significant improvement in SLE-APAH within a six-month period. ${ }^{36}$ Prompt treatment of $\mathrm{PAH}$ with newer PAH therapy as well as immunosuppression can reduce morbidity and prolong survival. ${ }^{34}$ Therefore, PAH-targeted treatment, such as prostacyclin analogs, endothelin receptor antagonists, and phosphodiesterase -5 inhibitors, and immunosuppressive therapies should be considered.

The limitation of this retrospective study was that there was no RHC data. Hence, patients with SLE along with suspected PAH screened by echocardiography needs to be undergo RHC.

In conclusion, in this study, we investigated the frequency and risk factors of SLE-associated PH in Chinese population to obtain the convenient and simple test items, which can provide evidence for early diagnosis. Patients with SLE who manifest tachypnea, Raynaud's phenomenon, serositis, epilepsy and positive anti-U1RNP are at high risk of $\mathrm{PH}$ complication. These patients should be given regular examination through echocardiography in addition to monitoring of lupus disease activity, especially for SLE survivors with high SDI score, to decrease the probability of developing cardiovascular diseases, including $\mathrm{PH}$, and improve survival rate and quality of life.

\section{Declaration of conflicting interests}

The authors declared no conflicts of interest with respect to the authorship and/or publication of this article.

\section{Funding}

The authors received no financial support for the research and/or authorship of this article.

\section{REFERENCES}

1. Simonneau G, Gatzoulis MA, Adatia I, Celermajer D, Denton C, Ghofrani A, et al. Updated clinical classification of pulmonary hypertension. J Am Coll Cardiol 2013;62:34-41. 
2. Zhao J, Wang Q, Liu Y, Tian Z, Guo X, Wang H, et al. Clinical characteristics and survival of pulmonary arterial hypertension associated with three major connective tissue diseases: A cohort study in China. Int J Cardiol 2017;236:432-7.

3. Bazan IS, Mensah KA, Rudkovskaia AA, AdontengBoateng P, Herzog EL, Buckley L, et al. Pulmonary arterial hypertension in the setting of scleroderma is different than in the setting of lupus: A review. Respir Med 2018;134:42-6.

4. Dhala A. Pulmonary arterial hypertension in systemic lupus erythematosus: current status and future direction. Clin Dev Immunol 2012;2012:854941.

5. Chung L, Liu J, Parsons L, Hassoun PM, McGoon M, Badesch DB, et al. Characterization of connective tissue disease-associated pulmonary arterial hypertension from REVEAL: identifying systemic sclerosis as a unique phenotype. Chest 2010;138:1383-94.

6. Jais X, Launay D, Yaici A, Le Pavec J, Tchérakian $\mathrm{C}$, Sitbon $\mathrm{O}$, et al. Immunosuppressive therapy in lupus- and mixed connective tissue disease-associated pulmonary arterial hypertension: a retrospective analysis of twenty-three cases. Arthritis Rheum 2008;58:521-31.

7. Qian J, Wang Y, Huang C, Yang X, Zhao J, Wang $\mathrm{Q}$, et al. Survival and prognostic factors of systemic lupus erythematosus-associated pulmonary arterial hypertension: A PRISMA-compliant systematic review and meta-analysis. Autoimmun Rev 2016;15:250-7.

8. O'Byrne ML, Kennedy KF, Kanter JP, Berger JT, Glatz AC. Risk Factors for Major Early Adverse Events Related to Cardiac Catheterization in Children and Young Adults With Pulmonary Hypertension: An Analysis of Data From the IMPACT (Improving Adult and Congenital Treatment) Registry. J Am Heart Assoc 2018;7. pii: e008142.

9. D'Alto M, Romeo E, Argiento P, Di Salvo G, Badagliacca R, Cirillo AP, et al. Pulmonary arterial hypertension: the key role of echocardiography. Echocardiography 2015;32 Suppl 1:S23-37.

10. Ruiz-Irastorza G, Garmendia M, Villar I, Egurbide MV, Aguirre C. Pulmonary hypertension in systemic lupus erythematosus: prevalence, predictors and diagnostic strategy. Autoimmun Rev 2013;12:410-5.

11. Petri M, Orbai AM, Alarcón GS, Gordon C, Merrill JT, Fortin PR, et al. Derivation and validation of the Systemic Lupus International Collaborating Clinics classification criteria for systemic lupus erythematosus. Arthritis Rheum 2012;64:2677-86.

12. Abbas AE, Fortuin FD, Schiller NB, Appleton CP, Moreno CA, Lester SJ. Echocardiographic determination of mean pulmonary artery pressure. Am J Cardiol 2003;92:1373-6.

13. Bombardier C, Gladman DD, Urowitz MB, Caron $\mathrm{D}$, Chang $\mathrm{CH}$. Derivation of the SLEDAI. A disease activity index for lupus patients. The Committee on Prognosis Studies in SLE. Arthritis Rheum 1992;35:630-40.
14. Gladman D, Ginzler E, Goldsmith C, Fortin P, Liang M, Urowitz $\mathrm{M}$, et al. The development and initial validation of the Systemic Lupus International Collaborating Clinics/American College of Rheumatology damage index for systemic lupus erythematosus. Arthritis Rheum 1996;39:363-9.

15. Li M, Wang Q, Zhao J, Li Z, Ye Z, Li C, et al. Chinese SLE Treatment and Research group (CSTAR) registry: II. Prevalence and risk factors of pulmonary arterial hypertension in Chinese patients with systemic lupus erythematosus. Lupus 2014;23:1085-1.

16. Janda S, Shahidi N, Gin K, Swiston J. Diagnostic accuracy of echocardiography for pulmonary hypertension: a systematic review and meta-analysis. Heart 2011;97:612-22.

17. Kaymaz C, Akbal OY, Hakgor A, Tokgoz HC, Tanboga IH, Aktemur T, et al. Reappraisal of the reliability of Doppler echocardiographic estimations for mean pulmonary artery pressure in patients with pulmonary hypertension: a study from a tertiary centre comparing four formulae. Pulm Circ 2018;8:2045894018762270.

18. McLaughlin VV, Archer SL, Badesch DB, Barst RJ, Farber HW, Lindner JR, et al. ACCF/AHA 2009 expert consensus document on pulmonary hypertension a report of the American College of Cardiology Foundation Task Force on Expert Consensus Documents and the American Heart Association developed in collaboration with the American College of Chest Physicians; American Thoracic Society, Inc.; and the Pulmonary Hypertension Association. J Am Coll Cardiol 2009;53:1573-619.

19. McGoon MD, Miller DP. REVEAL: a contemporary US pulmonary arterial hypertension registry. Eur Respir Rev 2012;21:8-18.

20. Lian F, Chen D, Wang Y, Ye Y, Wang X, Zhan Z, et al. Clinical features and independent predictors of pulmonary arterial hypertension in systemic lupus erythematosus. Rheumatol Int 2012;32:1727-31.

21. Tselios K, Gladman DD, Urowitz MB. Systemic lupus erythematosus and pulmonary arterial hypertension: links, risks, and management strategies. Open Access Rheumatol 2016;9:1-9.

22. Huber LC, Bye $\mathrm{H}$, Brock M. The pathogenesis of pulmonary hypertension--an update. Swiss Med Wkly 2015;145:14202.

23. Yokoi T, Tomita Y, Fukaya M, Ichihara S, Kakudo $\mathrm{K}$, Takahashi Y. Pulmonary hypertension associated with systemic lupus erythematosus: predominantly thrombotic arteriopathy accompanied by plexiform lesions. Arch Pathol Lab Med 1998;122:467-70.

24. Duong HT, Erzurum SC, Asosingh K. Pro-angiogenic hematopoietic progenitor cells and endothelial colony-forming cells in pathological angiogenesis of bronchial and pulmonary circulation. Angiogenesis 2011;14:411-22.

25. Prete M, Fatone MC, Vacca A, Racanelli V, Perosa F. Severe pulmonary hypertension as the initial 
manifestation of systemic lupus erythematosus: a case report and review of the literature. Clin Exp Rheumatol 2014;32:267-74.

26. La Paglia GMC, Leone MC, Lepri G, Vagelli R, Valentini E, Alunno A, et al. One year in review 2017: systemic lupus erythematosus. Clin Exp Rheumatol 2017;35:551-61.

27. Nickel NP, O'Leary JM, Brittain EL, Fessel JP, Zamanian RT, West JD, et al. Kidney dysfunction in patients with pulmonary arterial hypertension. Pulm Circ 2017;7:38-54.

28. Hao YJ, Jiang X, Zhou W, Wang Y, Gao L, Wang Y, et al. Connective tissue disease-associated pulmonary arterial hypertension in Chinese patients. Eur Respir J 2014;44:963-72.

29. Benza RL, Miller DP, Gomberg-Maitland M, Frantz RP, Foreman AJ, Coffey CS, et al. Predicting survival in pulmonary arterial hypertension: insights from the Registry to Evaluate Early and Long-Term Pulmonary Arterial Hypertension Disease Management (REVEAL). Circulation 2010;122:164-72.

30. Chung SM, Lee CK, Lee EY, Yoo B, Lee SD, Moon HB. Clinical aspects of pulmonary hypertension in patients with systemic lupus erythematosus and in patients with idiopathic pulmonary arterial hypertension. Clin Rheumatol 2006;25:866-72.

31. Mazurek JA1, Forfia PR. Enhancing the accuracy of echocardiography in the diagnosis of pulmonary arterial hypertension: looking at the heart to learn about the lungs. Curr Opin Pulm Med 2013;19:437-45.

32. Crowe T, Jayasekera G, Peacock AJ. Non-invasive imaging of global and regional cardiac function in pulmonary hypertension. Pulm Circ 2018;8:2045893217742000.

33. Ghofraniha L, Mirfeizi Z, Khabbaz FS, Vakilian F, Eslami S. Correlation of echocardiographic findings of pulmonary hypertension with six-minute walk test and plasma pro b-type natriuretic peptide level in systemic lupus erythematous. Electron Physician 2017;9:5122-8.

34. Prabu A, Gordon C. Pulmonary arterial hypertension in SLE: what do we know? Lupus 2013;22:1274-85.

35. Yasuoka H, Shirai Y, Tamura Y, Takeuchi T, Kuwana M. Predictors of Favorable Responses to Immunosuppressive Treatment in Pulmonary Arterial Hypertension Associated With Connective Tissue Disease. Circ J 2018;82:546-54.

36. Kommireddy S, Bhyravavajhala S, Kurimeti K, Chennareddy S, Kanchinadham S, Rajendra Vara Prasad I, et al. Pulmonary arterial hypertension in systemic lupus erythematosus may benefit by addition of immunosuppression to vasodilator therapy: an observational study. Rheumatology (Oxford) 2015;54:1673-9. 